\title{
Effect of body position on measurements of diffusion capacity after exercise
}

\author{
Ian B Stewart, Jim E Potts, Don C McKenzie, Ken D Coutts
}

\begin{abstract}
Background-Pulmonary diffusing capacity for carbon monoxide (Dlco), alveolar capillary membrane diffusing capacity (Dm), and pulmonary capillary blood volume (Vc) are all significantly reduced after exercise.

Objective-To investigate whether measurement position affects this impaired gas transfer.

Methods-Before and one, two, and four hours after incremental cycle ergometer exercise to fatigue, single breath Dlco, Dm, and Vc measurements were obtained in 10 healthy men in a randomly assigned supine and upright seated position.

Results-After exercise, Dlco, Dm, and Vc were significantly depressed compared with baseline in both positions. The supine position produced significantly higher values over time for Dlco (5.22 (0.13) $v 4.66$ $(0.15) \mathrm{ml} / \mathrm{min} / \mathrm{mm} \mathrm{Hg} / \mathbf{l}, \mathrm{p}=0.022)$ and $\mathrm{Dm}$ (6.78 (0.19) v $6.03(0.19) \mathrm{ml} / \mathrm{min} / \mathrm{mm} \mathrm{Hg} / 1$, $p=0.016)$, but there was no significant position effect for Vc. There was a similar pattern of change over time for Dlco, $\mathrm{Dm}$, and $\mathrm{Vc}$ in the two positions.

Conclusions-The change in Dlco after exercise appears to be primarily due to a decrease in Vc. Although the mechanism for the reduction in Vc cannot be determined from these data, passive relocation of blood to the periphery as the result of gravity can be discounted, suggesting that active vasoconstriction of the pulmonary vasculature and/or peripheral vasodilatation is occurring after exercise.

(Br F Sports Med 2000;34:440-444)
\end{abstract}

Keywords: pulmonary; diffusing capacity; exercise; body position; blood volume; lung

University of British Columbia, Allan McGavin Sports Medicine Center, John Owen Pavilion, 3055

Wesbrook Mall,

Vancouver, British

Columbia, V6T 1Z3,

Canada

I B Stewart

J E Potts

D C McKenzie

K D Coutts

Correspondence to:

I B Stewart

email:

kiwi@interchange.ubc.ca

Accepted for publication 20 April 2000
The effect of changing the body position from seated to supine is to increase the diffusing capacity for carbon monoxide of the lung (Dlco). This increase has traditionally been attributed to an increase in the pulmonary capillary blood volume (Vc) in the upper pulmonary lobes. ${ }^{1}$ However, the capillaries in the upper regions of the lung are not fully recruited because of the relatively low vascular pressure. Thus, although Vc is substantially elevated, the rise in the alveolar capillary membrane diffusing capacity (Dm) is insignificant. ${ }^{2}$

Dlco is impaired after exercise of different durations and intensities. ${ }^{3-5}$ The restriction of Dlco after exercise persists for at least six hours and may not return to baseline values for one to two days. ${ }^{6-8}$ It reflects a decrease in both Dm and Vc. ${ }^{56}$
Blood pooling in the periphery after exercise and therefore compensatory shunting, as transient changes in venous tone in both pulmonary and systemic circulations direct blood flow away from the thorax to clear the metabolic byproducts of exercise, have been suggested to explain the decrement in Vc. ${ }^{6} 9$ Thoracic electrical impedance is elevated after exercise, indicating a lower thoracic fluid volume $^{811}$ and supporting the theory about reduced central blood volume.

Microvascular injury to the alveolar capillary membrane resulting in acute subclinical interstitial pulmonary oedema has been suggested to be a mechanism for the reduced Dm. ${ }^{3}{ }^{12-14}$ Maximal hydrostatic pressures in human pulmonary capillaries are not accurately known, although they are estimated to be in the region of $30-40 \mathrm{~mm} \mathrm{Hg} .{ }^{15}$ These pressures are in the range where stress failure of the pulmonary capillaries has been demonstrated in experimental animals. ${ }^{12}{ }^{16}$ When pressures this high develop, the vascular endothelium may be injured allowing the movement of fluid from the vascular space to the interstitium of the lung. Pulmonary oedema could also result from an increased blood volume in the distended lung capillaries increasing permeability and promoting fluid shifts. ${ }^{7}$ An associated inability of the pulmonary lymphatic system to clear the accumulated fluid may explain the depressed Dm after exercise.$^{17}$

If a change in body position from seated to supine at rest results in increased venous return and thereby promotes an increase in blood in the pulmonary capillaries, then a supine body position after exercise should also promote a reduced Vc. Hanel et $a l,{ }^{9}$ investigating the redistribution of blood from the central vascular bed to more distal regions after exercise, reported a decrease in Dlco in both seated and supine positions ( $6 \%$ and $12 \%$ respectively). ${ }^{9} \mathrm{~A}$ corresponding change in thoracic to thigh electrical impedance ratio $(+14 \%)$, in the seated position only, supported the authors' hypothesis explaining the decrease in Dlco after exercise. The seated and supine measurements were conducted in the same testing session, separated by only 15 minutes, despite the recovery being solely in a supine position.

The independent changes in Dlco associated with a change in body position and recovery from exercise have been confirmed on many occasions, ${ }^{2818}$ but the interaction of these two treatments remains unresolved and forms the purpose of this investigation. If the decrease in Dlco and Dm after exercise were a function of a relocation of blood into the periphery causing a decrease in Vc, it was hypothesised that placing the subject in a supine position would 
enhance venous return after exercise and limit the decrease in central blood volume. This would return Vc toward baseline supine levels compared with the seated levels which would remain depressed.

\section{Methods}

Ten healthy non-smoking men were recruited. All were required to have normal pulmonary function with no known history of cardiovascular or respiratory disease. Before any testing, subjects received a verbal description of the experiment and completed a written consent form. This study was approved by the clinical screening committee for research and other studies involving human subjects of the University of British Columbia.

EXPERIMENTAL PROTOCOL

Subjects were required to report to the laboratory on two separate occasions, separated by at least 72 hours. Each testing session followed a similar protocol. To stabilise the pulmonary system, subjects were required to rest in their assigned position for 15 minutes before the measurement of Dlco. ${ }^{19}$ Pulmonary function was assessed by a Collins survey tach pulmonary function testing unit (Collins DS/PLUS II; Warren E Collins Inc, Baintree, Massachusetts, USA). Baseline values of Dlco were also obtained. A maximal cycle ergometer test was then undertaken. A total of five diffusion measurements were made, including one baseline measure and four measurements at 15 minutes, one, two, and four hours after the maximal cycle test. The two testing sessions differed only in the body position of the subject during the diffusion and pulmonary function measurements. Two positions were investigated, supine and seated. At all diffusion measurement periods, (a) heart rate was recorded using a telemetric monitor (Polar Vantage XL; Polar Electro Oy, Kempele, Finland) to evaluate a resting cardiovascular state, and $(b)$ haemoglobin was measured using a direct reading haemoglobinometer (HemoCue, Helsingborg, Sweden) to correct Dlco measures. ${ }^{17}$

MAXIMAL CYCLE ERGOMETER TEST

For the maximal cycle exercise test, subjects cycled on an electronically braked cycle ergometer (Quinton Excalibur; Lode, Groningen, The Netherlands) at a self selected cadence (about 30-120 rpm), against a workload increasing at $30 \mathrm{~W} / \mathrm{min}$ until volitional fatigue. Expired gases were collected through a two way non-rebreathing valve (HansRudolph, no 2700B, Kansas City, Missouri, USA) and analysed with an automated metabolic system (Rayfield System consisting of an airflow meter (Vacumetrics no 17150, Ventura, California, USA), and S-3A oxygen analyser and a carbon dioxide analyser (Ametek, Pittsburg, Pennsylvania, USA)). Before each testing session, the gas analysers were calibrated with a known gas mixture and room air. Heart rate was recorded every 15 seconds, and arterial oxygen saturation $\left(\mathrm{SaO}_{2}\right)$ was measured with a pulse ear oximeter (Ohmeda Biox 3740; BOC Health Care Inc, Edison, New Jersey, USA).

COLLECTION OF PULMONARY DIFFUSION DATA Pulmonary diffusing capacity was determined by the single breath method, ${ }^{20}$ which required the subjects to make a maximal inspiration from residual volume of a gas mixture containing $20.9 \% \mathrm{O}_{2}, 9.7 \% \mathrm{He}, 0.3 \% \mathrm{CO}$ balanced with $\mathrm{N}_{2}$. The breath was held for about 10 seconds, and then expired. The first litre of expired gas was discarded, and the next $750 \mathrm{ml}$ was considered to be an alveolar sample uncontaminated by dead space gas. Concentrations of $\mathrm{CO}$ were measured using an infrared analyser (type 101; Morgan, Kent, UK). Each diffusion measurement was examined to ensure that the inspired volume was at least $90 \%$ of the forced vital capacity, the total time of inspiration was less than two seconds, and breath hold time was between 9 and 11 seconds. ${ }^{21}$ Measurements were made in duplicate separated by five minutes to ensure elimination of the test gas from the lungs. Both tests were within $10 \%$ or $3 \mathrm{ml} / \mathrm{min} / \mathrm{mm} \mathrm{Hg}$ or a third test was performed. ${ }^{22}$ The average of the two closest values was recorded. The diffusion apparatus was calibrated daily for both volume and CO.

To calculate $\mathrm{Dm}$ and $\mathrm{Vc}$, a second Dlco (Dlco $90 \% \mathrm{O}_{2}$ ) test was performed. Subjects were required to breathe for five minutes through a low resistance valve (Hans Rudolph, no 2700B) attached to a Douglas bag filled with a gas mixture of about $90 \% \mathrm{O}_{2}$ and $10 \%$ $\mathrm{N}_{2}$. The Dlco $90 \% \mathrm{O}_{2}$ test was immediately performed in the same manner as the $21 \% \mathrm{O}_{2}$. The 10 second breath hold was of a gas mixture of $90 \% \mathrm{O}_{2}, 10 \% \mathrm{He}$, and $0.3 \% \mathrm{CO}$. Dm and Vc were then determined. ${ }^{20}$ The partitioning technique is consistent with that performed by numerous authors, ${ }^{56}$ and the reliability has been shown by test-retest correlation for both gas measures $\left(21 \%\right.$ and $\left.90 \% \mathrm{O}_{2}\right)$ to be $r=0.98$ and $r=0.96$ respectively. ${ }^{23}$

\section{STATISTICAL ANALYSIS}

Data were examined using a 2 (position) by 5 (time) factorial analysis of variance with repeated measures on both factors. Time effects were analysed using the Dunnet test for multiple comparisons to a control group, in which means after exercise were compared with resting values. If a significant interaction occurred, Scheffe's post hoc procedure was applied for further comparison. Student's $t$ tests were used to compare data for resting pulmonary function and other descriptive data in both positions. The level of significance was $\mathrm{p}<0.05$ for all statistical comparisons.

\section{Results}

Ten healthy men completed the study (mean (SD) age 22.3 (2.4) years, height 180.3 (8.9) $\mathrm{cm}$, and weight $80.4(8.6) \mathrm{kg}$ ). Data on resting respiratory function showed no signs of abnormality (table 1). 
Table 1 Resting pulmonary function data for subjects in the two measurement positions

\begin{tabular}{lll}
\hline & Seated $(n=10)$ & Supine $(n=10)$ \\
\hline FVC (1) & $5.59(0.29)$ & $5.16(0.27)^{\star}$ \\
FEV $_{1}(1)$ & $5.18(0.31)$ & $4.46(0.20)$ \\
FEV $_{1} /$ FVC $(\%)$ & $87(1.78)$ & $86(1.32)$ \\
$\mathrm{F}_{25-75 \%}(1 / \mathrm{s})$ & $5.46(0.25)$ & $4.87(0.19)^{\star}$ \\
PEFR $(1 / \mathrm{s})^{\star}$ & $10.35(0.58)$ & $9.34(0.59)^{\star}$
\end{tabular}

Values are means (SE).

* Significantly different from seated $(\mathrm{p}<0.05)$.

$\mathrm{FVC}$, forced vital capacity; $\mathrm{FEV}_{1}$, forced expiratory volume in one second; PEFR, peak expiratory flow rate.

Table 2 Metabolic variables during maximal cycle ergometer test

\begin{tabular}{|c|c|c|}
\hline & Session $1(n=10)$ & Session $2(n=10)$ \\
\hline $\mathrm{VO}_{2} \operatorname{MAX}(1 / \mathrm{min})$ & $4.26(0.12)$ & $4.22(0.13)$ \\
\hline $\mathrm{VO}_{2} \mathrm{MAX}(\mathrm{ml} / \mathrm{min} / \mathrm{kg})$ & $51.82(1.11)$ & $52.39(0.67)$ \\
\hline Peak power $(\mathrm{W})$ & $375(9.43)$ & $376(8.76)$ \\
\hline $\mathrm{SaO}_{2}(\%)$ at $\mathrm{VO}_{2} \mathrm{MAX}$ & $95.6(0.20)$ & $94.3(0.38)$ \\
\hline HRMAX (beats/min) & $191(2.75)$ & $188(3.26)$ \\
\hline
\end{tabular}

Values are means (SE).

$\mathrm{VO}_{2} \mathrm{MAX}$, Maximal oxygen consumption; $\mathrm{SaO}_{2}$, arterial oxyhaemoglobin saturation; HRMAX, maximal heart rate.

Table 3 Heart rate (beats/min) at rest (baseline) and 15 minutes and one, two, and four hours after maximal exercise measured in the seated or supine position

\begin{tabular}{lll}
\hline & Seated & Supine \\
\hline Baseline & $61(1.2)$ & $54(2.7)$ \\
$15 \mathrm{~min}$ & $88(2.8)^{\star}$ & $82(3.6)^{\star}$ \\
$1 \mathrm{~h}$ & $64(2.6)$ & $60(2.4)$ \\
$2 \mathrm{~h}$ & $58(1.7)$ & $53(1.6)$ \\
$4 \mathrm{~h}$ & $58(1.2)$ & $50(1.4)$
\end{tabular}

Values are means (SE).

^Significantly different from baseline $(\mathrm{p}<0.05)$.

MAXIMAL CYCLE ERGOMETER TESTS

Data for the two incremental ergometer tests to volitional fatigue showed that equal stress was applied to the subjects at each testing session (table 2). No subjects in this study were classified as having exercise induced hypoxaemia (an arterial oxygen saturation of less than $91 \%) .^{24}$

\section{CARDIOVASCULAR VARIABLES}

The significantly elevated heart rate $(46 \%)$ obtained immediately before the 15 minute Dlco determination indicated that, regardless of body position, the metabolic level in some subjects had not returned to that found before exercise (table 3). The 15 minute data for Dlco, $\mathrm{Dm}$, and Vc were therefore removed from the analyses, because a resting cardiovascular state was required for standardised comparisons. This resulted in a position effect becoming significant for Dlco $(\mathrm{p}=0.022 v \mathrm{p}=0.058$ for the full analysis). There was no other significant change in Dlco, Dm, or Vc, when compared with the full analysis.

PULMONARY DIFFUSION MEASUREMENTS

Dlco was significantly higher in the supine than the seated measurements $(5.22(0.13) v 4.66$ (0.15) $\mathrm{ml} / \mathrm{min} / \mathrm{mm} \mathrm{Hg} / \mathrm{l} ; \mathrm{p}=0.022)$. Both positions showed significant reductions in Dlco at one, two, and four hours after exercise (fig 1).

Vc and Dm were both significantly lower than baseline at one, two, and four hours after exercise (figs 2 and 3 respectively). Dm showed a body position effect, with the supine position

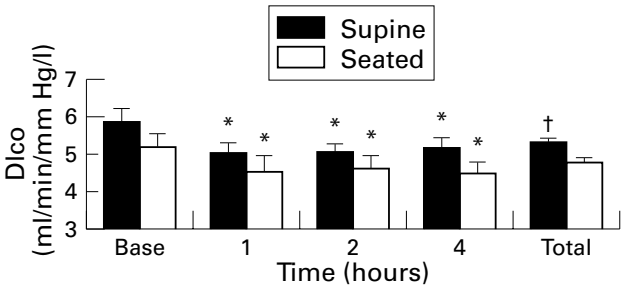

Figure 1 Pulmonary diffusing capacity for carbon monoxide (Dlco) corrected for alveolar volume at rest (Base), after exercise, and for each position regardless of time (Total). Values are means (SE). *Significantly lower than baseline value for the same position; tsignificantly higher than seated value; $p<0.05$.

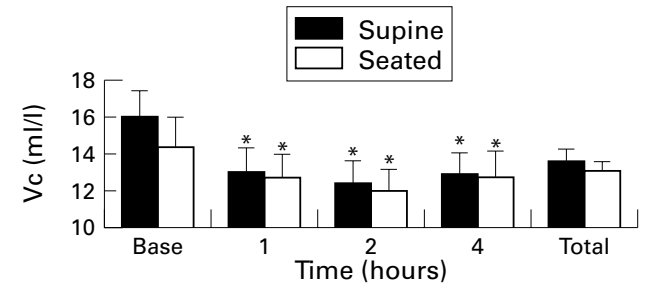

Figure 2 Pulmonary capillary blood volume (Vc) corrected for alveolar volume at rest (Base), after exercise, and for each position regardless of time (Total). Values are means (SE). *Significantly lower than baseline value for the same position; $p<0.05$.

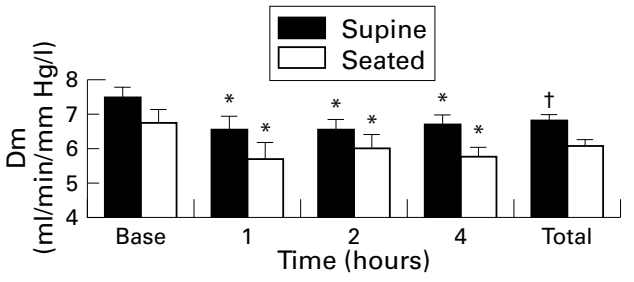

Figure 3 Membrane diffusing capacity (Dm) corrected for alveolar volume at rest (Base), after exercise, and for each position regardless of time (Total). Values are means (SE). *Significantly lower than for baseline value for the same position; tsignificantly higher than seated value; $p<0.05$.

producing higher values $(6.78(0.19) \approx 6.03$ (0.19) $\mathrm{ml} / \mathrm{min} / \mathrm{mm} \mathrm{Hg} / \mathrm{l}, \mathrm{p}=0.016)$. Vc was not significantly different between positions (13.49 (0.65) v $12.88(0.60) \mathrm{ml} / \mathrm{l}$, supine $v$ seated; $p=0.623)$. The two positions exhibited a similar pattern of change over the time periods for Dlco, Dm, and Vc.

\section{Discussion}

This study confirmed that pulmonary diffusing capacity is impaired during recovery from maximal exercise and that this reduction persists for at least four hours (fig 1 ).$^{6}{ }^{11}$ It also supports the recent finding that the impairment occurs in both a supine and upright seated position. ${ }^{9}$

PULMONARY CAPILLARY BLOOD VOLUME

Maximal exercise resulted in a reduced $\mathrm{Vc}$ in both a supine and upright seated position (fig 2). Position had no effect on the magnitude of the decrement over time, which was maintained four hours after exercise. The reduced $\mathrm{Vc}$ has been reported after exercise of various intensities, durations, and modalities..$^{3-5}{ }^{11}$ It has also been documented to remain depressed for a period of 24 hours. $^{67}$ 
It has been suggested that a reduced central blood volume is the primary mechanism for the impaired Dlco after exercise. ${ }^{911}$ The decreased Vc found in our study (fig 2) supports this theory. Thoracic fluid volume, measured by electrical impedance, is decreased after exercise and has been shown to be still decreased two to three hours after exercise. ${ }^{8911}$ A parallel relation between the decrease in Vc and an increase in thoracic electrical impedance indicates a reduction in central fluid volume, specifically blood. However, the mechanism behind this reduction in central blood volume remains unknown.

Inactivity for a prolonged period removes the effect of the muscle pump that assists venous return, suggesting that peripheral pooling of blood may be the cause of the decreased Vc after exercise. Even without a statistically significant position effect for Vc (fig 2), the result of the large variance in this measurement, the clear trend of the supine position causing larger falls in Vc from baseline $(-15.06 \%$ v $-9.74 \%)$ compared with the seated position suggests that a gravity induced relocation of blood to the peripheral circulation is not the cause of the reduced central blood volume and therefore the depressed Vc.

Other possible hypotheses include compensatory shunting consequent on heavy exercise, as transient changes in venous tone in both pulmonary and systemic circulations direct blood flow away from the thorax to clear metabolic waste products from exercised muscles. ${ }^{10}$ This seems unlikely as the development of a depressed Vc after only 10 minutes of cycling at $25 \% \mathrm{VO}_{2} \mathrm{MAX}^{5}$ and the increasing magnitude of the impairment up to six hours after exercise $^{6}$ do not reflect the time course of metabolic waste development and clearance respectively.

Increased thoracic electrical impedance, decreased levels of atrial natriuretic peptide, ${ }^{9}$ and decreased Vc all indicate a reduced central blood volume after exercise. The mechanism responsible for this cannot be determined from these data, although previous suggestions of passive redistribution of blood as the result of gravity can be discounted, suggesting that active vasoconstriction of the pulmonary vasculature and/or peripheral vasodilatation may be occurring after exercise.

ALVEOLAR CAPILLARY MEMBRANE DIFFUSING CAPACITY

After exercise, a decrease in Dm was observed (fig 3). This probably reflects an increase in the thickness of the alveolar capillary membrane, decreasing the rate of gas transfer between the alveolus and the red blood cells and/or a decrease in the available surface area for diffusion.

The decrease after exercise has been suggested to be an indicator of the development of acute pulmonary oedema. Direct attempts to quantify lung density and extravascular water content after exercise, using wet weight/dry weight ratio, indicator dilution, and computed tomography scanning techniques, have produced inconclusive results. ${ }^{35-27}$ The decreased
Dm one hour after 10 minutes of low intensity exercise ${ }^{5}$ and the lack of effect on $\mathrm{PaO}_{2}$ and performance during repeated bouts of exercise $^{1128}$ suggest that physiologically significant injury to the alveolar capillary membrane is not the mechanism responsible for the decreased Dlco and Dm after short duration maximal exercise.

It is also possible that the less depressed Dm in the supine position is the result of increased pulmonary lymph flow, which could serve to remove any accumulated interstitial fluid. Cardiac output has been found to be linearly related to pulmonary lymph flow. ${ }^{17}$ Coates et $a l^{17}$ suggested that the increase in perfused microvascular surface area with exercise was the primary determinant for the increase in pulmonary lymph flow. If such a relation exists after exercise, then the increase in perfused microvascular surface area in the supine position $^{29}$ may be expected to result in an increased rate of removal of any accumulated interstitial fluid.

The elevated $\mathrm{Dm}$ in the supine position compared with the upright seated position was probably due to gravity induced redistribution of blood from the distended capillaries in the lower zones of the lung, in the upright seated position, to the upper zones. This redistribution of blood could increase capillary recruitment and available surface area for diffusion in the supine position. The supine position decreased the ventilation to perfusion mismatch associated with an upright seated position. ${ }^{29}$ This increased uniformity in ventilation and perfusion in the supine position could increase Dm, through an increase in surface area available for gas transfer.

\section{SUMMARY}

This study confirms that Dlco is reduced after incremental cycling to volitional fatigue. It also indicates that the diffusion impairment is independent of measurement position. The change in Dlco appears to be primarily due to a decrease in Vc. The limited decrease in Dm in the supine position was probably due to redistribution of blood within the lung, as the result of gravity, increasing the surface area available for diffusion. The decrease in Vc has been previously attributed to reduced central blood volume. Although the mechanism for this reduction cannot be determined from these data, previous suggestions of passive relocation of blood to the periphery caused by gravity can be discounted, suggesting that active vasoconstriction of the pulmonary vasculature and/or peripheral vasodilatation may be occurring after exercise.

\footnotetext{
1 Newman F. The effect of a change in posture on alveolar-capillary diffusion and capillary blood volume in the human lung . F Physiol (Lond) 1962;162:29-30.

2 Chang SC, Chang HI, Liu SY, et al. Effects of body position and age on membrane diffusing capacity and pulmonary capillary blood volume. Chest 1992;102:139-42.

3 Caillaud C, Serre-Cousine O, Anselme F, et al. Computerized tomography and pulmonary diffusing capacity in highly trained athletes after performing a triathlon. $f \mathrm{Appl}$ highly trained athletes after

4 Manier G, Moinard J, Stoicheff H. Pulmonary diffusing capacity after maximal exercise. $\mathcal{F}$ Appl Physiol 1993;75: $2580-5$.
} 
5 Sharratt M, Potts J, McKenzie DC. The effect of exercise intensity on post-exercise measurements of lung diffusion [abstract]. Physiologist 1996;39:A47.

6 Sheel AW, Coutts KD, Potts JE, et al. The time course of pulmonary diffusing capacity changes following maxima exercise. Respir Physiol 1998;111:271-81.

7 Rasmussen BS, Elkjaer P, Juhl B. Impaired pulmonary and cardiac function after maximal exercise. F Sports Sci 1988; 6:219-28.

8 Rasmussen J, Hanel B, Saunamaki K, et al. Recovery of pulmonary diffusing capacity after maximal exercise. F Sports Sci 1992;10:525-31

9 Hanel B, Teunissen I, Rabol A, et al. Restricted postexercise pulmonary diffusion capacity and central blood volume depletion. F Appl Physiol 1997;83:11-17.

10 Lama IL, Potts J, Sheel AW, et al. Pulmonary diffusing capacity and exercise-induced hypoxemia in highly trained capacity and exercise-induced hypoxemia in

11 Hanel B, Clifford PS, Secher NH. Restricted postexercise pulmonary diffusion capacity does not impair maximal pulmonary diffusion capacity does not impair
transport for $\mathrm{O}_{2}$. F Appl Physiol 1994;77:2408-12.

12 West JB, Mathieu-Costello O. Structure, strength, failure, and remodeling of the pulmonary blood-gas barrier. Annu Rev Physiol 1999;61:543-72.

13 Hopkins SR, Schoene RB, Henderson WR, et al. Intense exercise impairs the integrity of the pulmonary blood-gas barrier in elite athletes. Am $\mathcal{f}$ Respir Crit Care Med 1997;155:1090-4

14 Hopkins SR, Schoene RB, Henderson WR, et al. Sustained submaximal exercise does not alter the integrity of the lung blood-gas barrier in elite athletes. F Appl Physiol 1998;84: 1185-9.

15 Wagner PD, Gale GE, Moon RE, et al. Mechanisms of exercise-induced hypoxemia in horses. 7 Appl Physiol 1986; 61:260-70.

16 Tsukimoto K, Mathieu-Costello O, Prediletto T, et al. Ultrastructural appearances of pulmonary capillaries at high transmural pressures. F Appl Physiol 1991;71:573-82.

17 Coates G, O'Brodovich H, Jefferies AL, et al. Effects of exercise on lung lymph flow in sheep and goats during normoxia and hypoxia. $\mathcal{F}$ Clin Invest 1984;74:133-41.

18 Miles DS, Doerr CE, Schonfeld SA, et al. Changes in pulmonary diffusing capacity and closing volume after running a marathon. Respir Physiol 1983;52:349-59.

19 Billiet L. Pulmonary diffusing capacity on exercise. Bern: Hans Ruber, 1971.

20 Roughton F, Forster RE. Relative importance of diffusion and chemical reaction rates in determining rate of exchange of gases in the human lung, with special reference to true diffusing capacity of the pulmonary membrane and volume of blood in the lung capillaries. F Appl Physiol 1957; 11:290-302.

21 Ogilvie CM, Forster RM, Blakemore WS, et al. Standardized breath holding technique for the clinical measurement of the diffusing capacity of the lung for carbon monoxide. 7 Clin Invest 1957;36:1-17.

22 American Thoracic Society. Single-breath carbon monoxide diffusing capacity. Am F Respir Crit Care Med 1995;152: 2185-98.

23 Sheel AW, Potts J, Lama IL, et al. Reliability of measurement of diffusing capacity of the pulmonary membrane and pulmonary capillary blood volume [abstract]. Physiologist 1996;39:A47.

24 Powers SK, Martin D, Dodd S. Exercise-induced hypoxemia in elite endurance athletes. Sports Med 1993;16:1422.

25 Marshall BE, Teichner RL, Kallos T, et al. Effects of posture and exercise on the pulmonary extravascular water volume in man. $\mathcal{F}$ Appl Physiol 1971;31:375-9.

26 Vaughan TR Jr, DeMarino E, Staub NC. Indicator dilution lung water and capillary blood volume in prolonged heavy exercise in normal men. Am Rev Respir Dis 1976;113:75762

27 Manier G, Duclos M, Arsac L, et al. Distribution of lung density after strenuous prolonged exercise. $\mathcal{F}$ Appl Physiol 1999;87:83-9.

28 McKenzie DC, Mayo JR, Potts J, et al. Changes in lung water content and pulmonary diffusion capacity following intense exercise in well-trained athletes [abstract]. Physiologist 1996;39:A47.

29 Brudin LH, Rhodes CG, Valind SO, et al. Interrelationships between regional blood flow, blood volume, and ventilation in supine humans. F Appl Physiol 1994;76:1205-10.

Take home message

The reduction in pulmonary diffusing capacity after exercise was not alleviated by altering body position, and was primarily due to a decrease in pulmonary capillary blood volume. The data indicate that the reduction in the latter was not due to passive relocation of blood, suggesting that active vasoconstriction of the pulmonary vasculature and/or peripheral vasodilatation may be occurring after exercise. 